\title{
Intra-night optical multiband variability of BL Lacertae during the 2011 outburst
}

\author{
M. Zhai ${ }^{1,2}$ and J. Y. Wei ${ }^{1}$ \\ 1 National Astronomical Observatories, Chinese Academy of Sciences, 100012 Beijing, PR China \\ e-mail: zm@nao.cas.cn \\ 2 Graduate University of Chinese Academy of Sciences, 100049 Beijing, PR China
}

Received 21 October 2011 / Accepted 21 November 2011

\section{ABSTRACT}

\begin{abstract}
Aims. We present our optical multiband ( $B$ and $R$ ) observations of BL Lacertae, which were acquired over 20 nights from May 26 to August 3, 2011, and analyse our data to study the optical emission variability properties of BL Lac.

Methods. The simultaneous photometric observations were carried out using the $0.8 \mathrm{~m}$ Tsinghua-NAOC Telescope (TNT) at the Xinglong Observatory of National Astronomical Observatories, Chinese Academy of Sciences (NAOC). TNT is equipped with the Princeton Instrument CCD and standard Johnson-Cousin UBVRI filters. Exposure times were well chosen to make a good compromise between high precision and high temporal density.

Results. A multipeak outburst was observed in 2011 observing season. The brightest magnitudes for BL Lac during our observations were $B=14.48 \mathrm{mag}$ and $R=12.89 \mathrm{mag}$ at $\mathrm{JD}=2455709.2810$ (May 27). One of the most rapid variations was detected on July 9 , when a flux increase of about $0.34 \mathrm{mag}$ occurred over $2.8 \mathrm{~h}$. In general, both the amplitude of variability and the variation rate are higher at the shorter wavelength. A colour analysis shows that the colour variability processes are apparently complicated. A bluerwhen-brighter trend was detected during the outburst. These trends may be the interactive contribution from a "mildly-chromatic" component and a "strongly-chromatic" one. Meanwhile, intra-night variability in $B$ and $R$ bands was found to be correlated, without any time delay exceeding 2.5 min.
\end{abstract}

Key words. galaxies: jets - quasars: general - galaxies: active - quasars: individual: BL Lacertae

\section{Introduction}

BL Lacertae (BL Lac; 1ES 2200+420; $z=0.0688 \pm 0.0002$ Miller \& Hawley 1977) is the prototype of the "BL Lac objects", which is one of the two blazar subclasses of active galactic nuclei (AGNs). BL Lacertae has been observed in the optical band since 1896 (Shen \& Usher 1970), and it is well known for its extreme variability at all wavelengths from longterm (months, years) oscillations to intra-day variability (IDV) (Villata et al. 2002). It is one of the best-studied objects in the optical and radio bands, and has been the favourite target of several campaigns by the Whole Earth Blazar Telescope (WEBT) collaboration since 1999 (Raiteri et al. 2010).

It is usually accepted that the central engine of AGNs is a supermassive black hole fed by infall of matter from an accretion disc, and the rotating and accreting black hole ejects a non-thermally emitting plasma jet that points close to the line of sight. Although the general framework seems to be well understood, the studies performed on the variation mechanism appear to be inconclusive. Furthermore, the violent flux variations observed in blazars have been accounted for by diverse models: shock-in-jet (e.g. Marscher 1996), changes in the Doppler factor (e.g. Dreissigacker \& Camenzind 1996; Villata \& Raiteri 1999), accretion disc instabilities (e.g. Wiita 1996), gravitational microlensing (e.g. Schneider \& Weiss 1987), etc. The shock-injet model suggests that the relativistic particle jets cause the IDV phenomenon even on the timescales of less than one hour (Gupta \& Joshi 2005; Zhai et al. 2011). The most rapid rate of change (as fast as $0.19 \mathrm{mag} / \mathrm{h}$ ) was detected by Villata et al. (2002). Their results from the analysis of the colour index indicated that the variability had two components, including inter-night mildly chromatic variations and strong bluer-whenbrighter chromatic intra-night flares, which may be the results of both intrinsic physical mechanism and changes in the Doppler factor for geometrical reasons (e.g. Villata et al. 2004).

Photometric observations can help in investigating their emissions and distinguishing among the various theoretical interpretations. To further study the BL Lac's multiband variability, colour behaviour, and the correlations among flux variations in different wavelengths, several long-term campaigns have been conducted to collect enormous optical-to-radio data by different research groups in the past decade. Noticeably, the source tended to be particularly variable during these campaigns.

Following the increase in the gamma-ray, optical, and nearinfrared activity of BL Lac reported in ATel 3368, 3371, and 3375 (Cutini 2011; Larionov et al. 2011; Carrasco et al. 2011), we initiated monitoring of the source using the $0.8 \mathrm{~m}$ Tsinghua-NAOC Telescope (TNT) at the Xinglong Observatory from May 31 until June 20, 2011. Following another bright flare from BL Lac detected by VERITAS and Fermi LAT on June 28 (Ong 2011; Gasparrini 2011), new observations were performed from July to Aug. 2011.

We performed the optical ( $B$ and $R$ ) monitoring of BL Lac during its summer outburst, in order to examine the characteristics of the shortest timescale variations of this source, and, especially, to search for any possible time delays between the bands. This paper is arranged as follows. Section 2 describes observations and the data analysis process, Sect. 3 presents the results and discussion, and the conclusions of the present work are reported in Sect. 4. 
Table 1. Complete log of observations of BL Lac.

\begin{tabular}{lccc}
\hline \hline Date (dd.mm.yyyy) & Filter & No. of data points & Duration $(\mathrm{h})$ \\
\hline 26.05 .2011 & $B R$ & $1 \times 2$ & 0.2 \\
27.05 .2011 & $B R$ & $1 \times 2$ & 0.2 \\
31.05 .2011 & $B R$ & $81 \times 2$ & 3.3 \\
01.06 .2011 & $B R$ & $96 \times 2$ & 3.1 \\
02.06 .2011 & $B R$ & $129 \times 2$ & 2.7 \\
03.06 .2011 & $B R$ & $29 \times 2$ & 0.8 \\
04.06 .2011 & $R$ & 191 & 3.6 \\
06.06 .2011 & $B R$ & $173 \times 2$ & 3.7 \\
08.06 .2011 & $B R$ & $1 \times 2$ & 0.1 \\
11.06 .2011 & $B R$ & $1 \times 2$ & 0.1 \\
12.06 .2011 & $B R$ & $1 \times 2$ & 0.1 \\
13.06 .2011 & $B R$ & $72 \times 2$ & 3.7 \\
17.06 .2011 & $B R$ & $48 \times 2$ & 3.1 \\
24.06 .2011 & $B R$ & $1 \times 2$ & 0.1 \\
25.06 .2011 & $B R$ & $1 \times 2$ & 0.1 \\
03.07 .2011 & $B R$ & $1 \times 2$ & 0.2 \\
08.07 .2011 & $B R$ & $197 \times 2$ & 5.5 \\
09.07 .2011 & $B R$ & $167 \times 2$ & 5.6 \\
31.07 .2011 & $R$ & 4 & 0.4 \\
03.08 .2011 & $B R$ & $52 \times 2$ & 2.1 \\
\hline Total 20 nights & & 2299 & 38.7 \\
\hline
\end{tabular}

\section{Observations and data reduction}

The simultaneous $(\Delta t \leqslant 2.5 \mathrm{~min}$ in most cases) photometric observations of BL Lac in Johnson-Cousin $B$ and $R$ passbands were alternately carried out using the TNT, which is an equatorial-mounted Cassegrain system with an $f / 10$ beam. It uses the Princeton Instrument $1340 \times 1300$ thin back-illuminated CCD, which has a pixel size of $\sim 20 \mu \mathrm{m}$ and is cooled by liquid nitrogen. The read-out noise and gain of the CCD were five electrons and 2.3 electrons/ADU, respectively. The entire CCD chip covers $\sim 11 \times 11 \operatorname{arcmin}^{2}$ of the sky, equipped with standard Johnson-Cousin $U B V R I$ filters in a filterwheel (Zhai et al. 2011). Exposure times were well chosen to lead to a good compromise between high precision and high temporal density, and the range was 25 to $150 \mathrm{~s}$ in $B$ band and 5-30 s in $R$ band. BL Lac was monitored as continuously as possible each observation night, and was observed more than ten clear nights throughout the observing run (see Table 1).

The raw data were bias and flat-field corrected using IRAF $^{1}$ software. No dark frame was needed since the CCD camera was cooled sufficiently. Instrumental magnitudes of BL Lac and comparison stars B and C from Smith et al. (1985) were extracted using an auto photometry package (AUTOPHOT), which was developed to minimize the repetition. Subsequently, the instrumental magnitudes were converted to apparent magnitudes through differential photometry. The star $\mathrm{C}$ was fainter than BL Lac in most cases during the observations, thus the rms errors are calculated from the star $\mathrm{C}$ using the formula

$\sigma=\sqrt{\frac{\sum\left(m_{\mathrm{c}}-\overline{m_{\mathrm{c}}}\right)^{2}}{N-1}}$

where $m_{\mathrm{c}}$ is the differential magnitude of star $\mathrm{C}$, while $\overline{m_{\mathrm{c}}}$ is the differential magnitude averaged over the entire dataset, and $N$ is the number of the observations on a given night. According to Hu et al. (2006) and Capetti et al. (2010), the host galaxy has similar colours to BL Lac. Thus, the contribution of the host galaxy was not subtracted from the total flux densities in our

\footnotetext{
${ }^{1}$ IRAF is distributed by NOAO, which is operated by AURA, Inc., under cooperative agreement with the NSF.
}

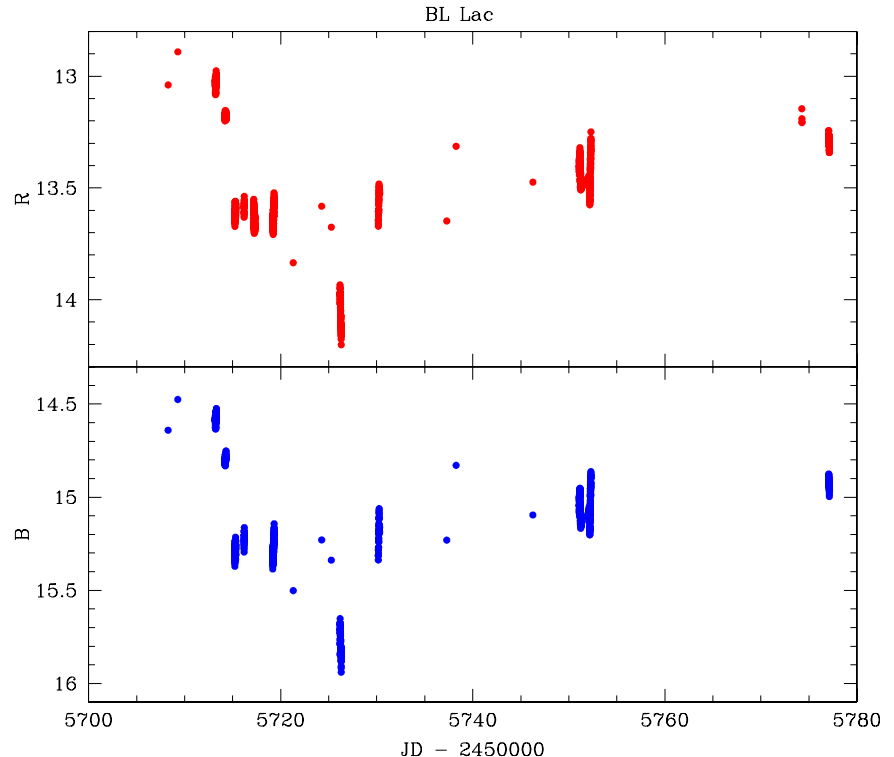

Fig. 1. Multiband light curves of BL Lac at $B$ (bottom panel) and $R$ (top panel) bands from TNT.

analysis, because it is also negligible for our purposes. The overall average error rms of photometry was not more than $0.01 \mathrm{mag}$.

\section{Results and discussion}

\subsection{Light curves}

Over our 2011 observing season, the BL Lac underwent some flares, which were partly monitored in the $B$ and $R$ bands by the TNT. Noticeable episodes of rapid variability were detected, including flux changes of several tenths of mag in a few hours. The overall $B$ and $R$ bands light curves obtained from May 26 to August 3, 2011 are plotted together in Fig. 1 with a total of 2299 data points. The maximum magnitude variations are $\triangle B=1.46 \mathrm{mag}$ and $\Delta R=1.31 \mathrm{mag}$. It is a pity that we did not completely monitor these flares because of the weather conditions or other campaigns.

A target-of-opportunity (ToO) observation was carried out owing to the Astronomer's Telegram of increased activity of BL Lac since May 26. An increasing gamma-ray flux from BL Lac was observed by the Fermi LAT on May 20, 2011 (Cutini 2011). Meanwhile, observations in St. Petersburg showed $R=13.87$ on May $14.99, R=13.06$ on May 21.95, and $R=12.71$ on May 22.97, with a variation of $0.36 \mathrm{mag}$ in $24.5 \mathrm{~h}$. It is important that these brightness levels are comparable to those reached during the outbursts of 1997-2004 (Larionov et al. 2011). During our observation, the object was indeed bright and reached $B=14.64$ and $R=13.04$ on May 26 $(\mathrm{JD}=2455$ 708.2740). As shown in Fig. 1, BL Lac was brighter on May $27(\mathrm{JD}=2455709.2810)$ and reached $B=14.48$ and $R=12.89$, which are the brightest magnitudes of BL Lac over our 2011 observing season.

The formal multiband monitoring began on May 31. The light curves of $B$ and $R$ bands for individual periods are displayed in Figs. 2-9. The magnitude of the source quickly dropped about $0.85 \mathrm{mag}$ in $B$-band, and $0.70 \mathrm{mag}$ in $R$-band from $\mathrm{JD}=2455713$ to 2455715 (Figs. 2-4). After some relatively stable periods, it reached a minimum on JD $=2455726.3(B=$ $15.94 \mathrm{mag}$ and $R=14.20 \mathrm{mag}$, see Fig. 6). The source brightness then rose rapidly in four days (see Fig. 7). VERITAS and 


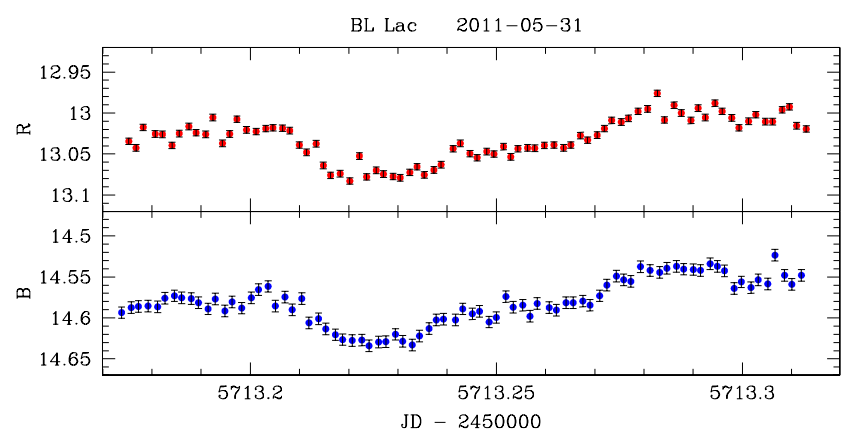

Fig. 2. Multiband light curves of BL Lac at $B$ (bottom panel) and $R$ (top panel) bands from TNT on May 31, 2011 (JD = 2455 713.1741$2455713.3123)$.

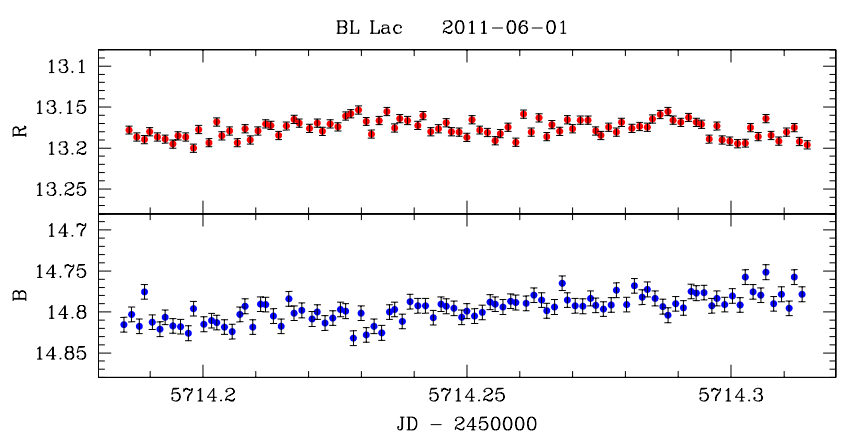

Fig. 3. Similar to Fig. 2, but with time on June 1, 2011 (JD = 2455 714.1851-2 455714.3140$)$.

Fermi LAT detected another bright flare on June 28 (Ong 2011; Gasparrini 2011), but it was cloudy here, so we had to follow up the new flare after July 3. BL Lac remained active until the end of our observations. Clear intra-night variations can be seen during the whole monitoring campaign. We detected a significant brightness change on July 9 (JD $=2455752.1856-$ 2455752.3051 , see Fig. 9) with $B$ and $R$ flux increase of about $0.34 \mathrm{mag}$ and $0.33 \mathrm{mag}$ in $2.8 \mathrm{~h}$, respectively.

We suggest that these flares are most likely to be real variations in the jet emission. It has been well accepted that intranight variability in radio-loud AGNs could be explained by the shock-in-jet model. The model suggests that the particles are accelerated by the shocks advancing down the jet. The overdense magnetic field may also enhance the optical synchrotron emission (Hu et al. 2006). Meanwhile, a rotating helical path in a curved jet has been used to explain the optical and radio behaviour of BL Lac in the past forty years by Villata et al. (2009). If the jet has a helical structure, different portions of the jet may be well aligned with the line of sight, which could cause temporal changes in the Doppler factor (Raiteri et al. 2009).

\subsection{Correlated variability}

The determination of time lag between two sets of data in different wavebands can be used to study the conditions in the inner regions of blazars. The time lag can be searched for using the discrete correlation function (DCF) (Edelson \& Krolik 1988; Hufnagel \& Bregman 1992; Fan et al. 2001). Firstly, we calculated the set of unbinned discrete correlations (UDCF) between these two data sets:

$$
U D C F_{i j}=\frac{\left(a_{i}-\bar{a}\right) \times\left(b_{j}-\bar{b}\right)}{\sqrt{\sigma_{a}^{2} \times \sigma_{b}^{2}}},
$$

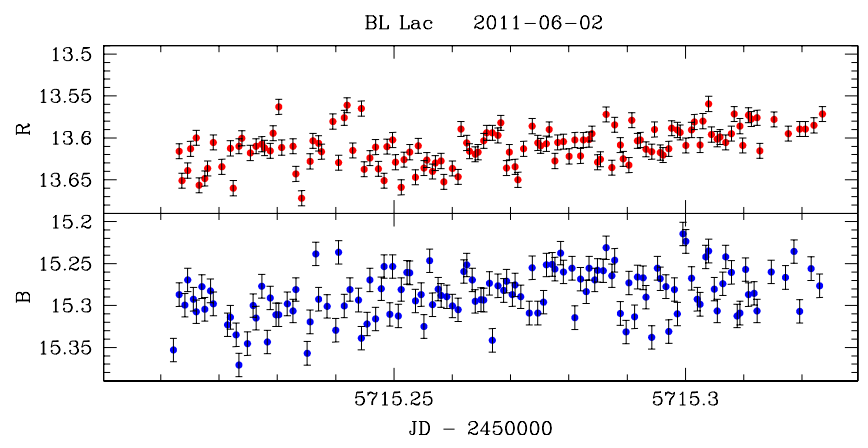

Fig. 4. Similar to Fig. 2, but with time on June 2, 2011 $(\mathrm{JD}=2455715.2128-2455715.3234)$.

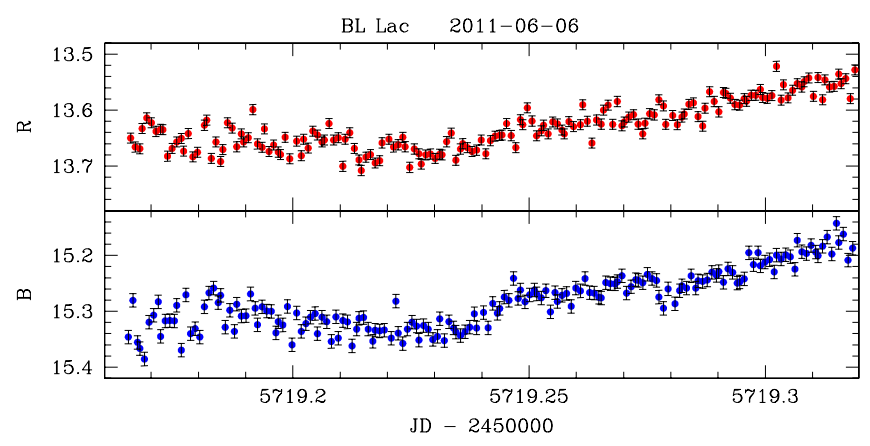

Fig. 5. Similar to Fig. 2, but with time on June 6, 2011 $(\mathrm{JD}=2455719.1650-2455719.3189)$.

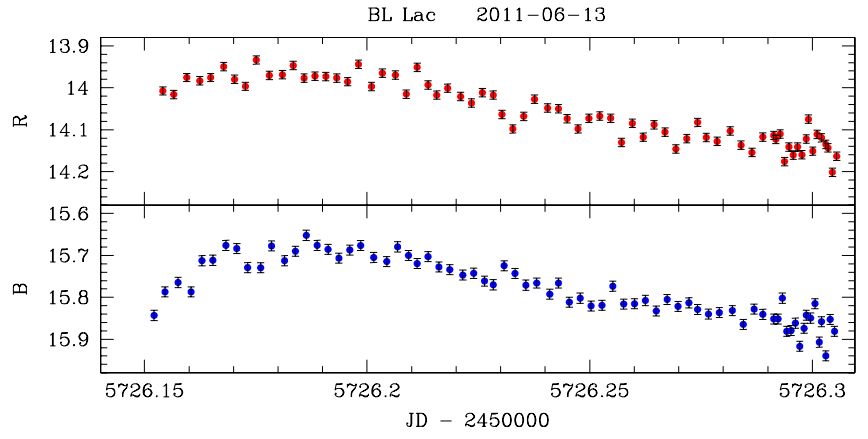

Fig. 6. Similar to Fig. 2, but with time on June 13, 2011 $(\mathrm{JD}=2455726.1525-2455726.3055)$.

where $a_{i}$ and $b_{j}$ are points in the data sets, $\bar{a}$ and $\bar{b}$ are the average values of the data sets, and $\sigma_{a}$ and $\sigma_{b}$ are the standard deviations in each data set.

Secondly, binning the $U D C F_{i j}$ in suitably sized time-bins in order to get the $D C F(\tau)$ for each time lag $\tau$ :

$D C F(\tau)=\frac{1}{M} \sum U D C F_{i j}(\tau)$,

where $M$ is the total number of pairs.

BL Lac was observed in $B$ and $R$ bands continuously and intensively on May 31, June 6, July 8, and July 9, for 3.3, $3.7,5.5$, and $5.6 \mathrm{~h}$, respectively. It is possible for us to discuss the correlated variability at high time-resolution in these four segments of data. Figure 11 shows that the $B$-band variation is correlated with the $R$-band variation. The location of the peak $\tau_{\text {peak }}$ and the corresponding correlation coefficient $r_{\text {peak }}$ are shown in Table 2. Peterson et al. (1998) recommended using the centroid of the DCF, $\tau_{\text {cent }}$ rather than $\tau_{\text {peak }}$, which cannot be interpreted as a physical quantity in any simple manner 
Table 2. Cross-correlation results (time unit: minutes).

\begin{tabular}{lcccccc}
\hline \hline Date (dd.mm.yyyy) & $\tau_{\text {peak }}$ of DCF & $r_{\text {peak }}$ & $\tau_{\text {peak }}$ of MC & $\tau_{\text {cent }}$ of DCF & $\tau_{\text {cent }}$ of MC & Time-resolution \\
\hline 31.05 .2011 & -2.5 & 0.906 & $-1.6 \pm 3.3$ & -1.3 & $-1.2 \pm 2.0$ & 2.5 \\
06.06 .2011 & 0.0 & 0.823 & $-1.2 \pm 1.7$ & -1.0 & $0.3 \pm 1.1$ & 1.3 \\
08.07 .2011 & -1.7 & 0.948 & $2.1 \pm 1.7$ & 0.1 & $0.6 \pm 0.9$ & 1.7 \\
09.07 .2011 & 0.0 & 0.970 & $0.0 \pm 0.0$ & 1.6 & $1.6 \pm 0.5$ & 1.7 \\
\hline
\end{tabular}

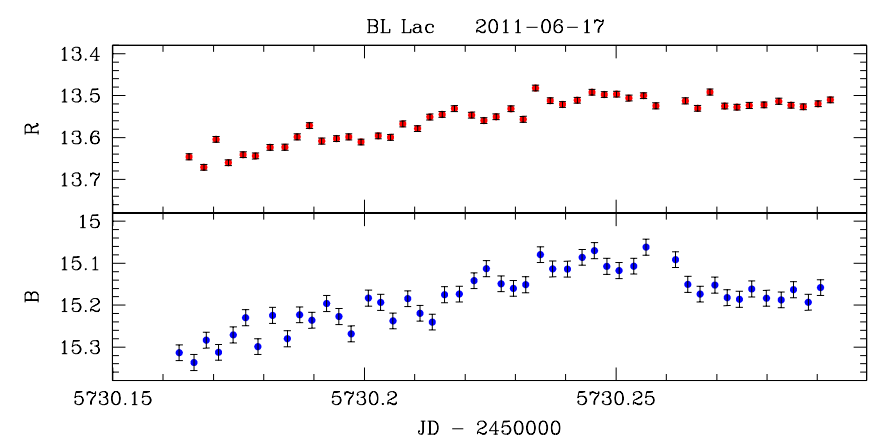

Fig. 7. Similar to Fig. 2, but with time on June 17, 2011 $(\mathrm{JD}=2455730.1632-2455730.2926)$.

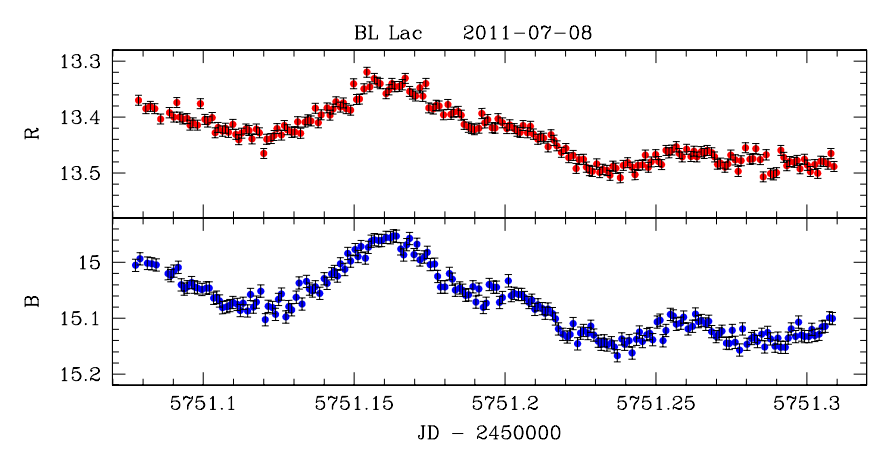

Fig. 8. Similar to Fig. 2, but with time on July 8, 2011 (JD = 2455 751.0779-2 455751.3090 ).

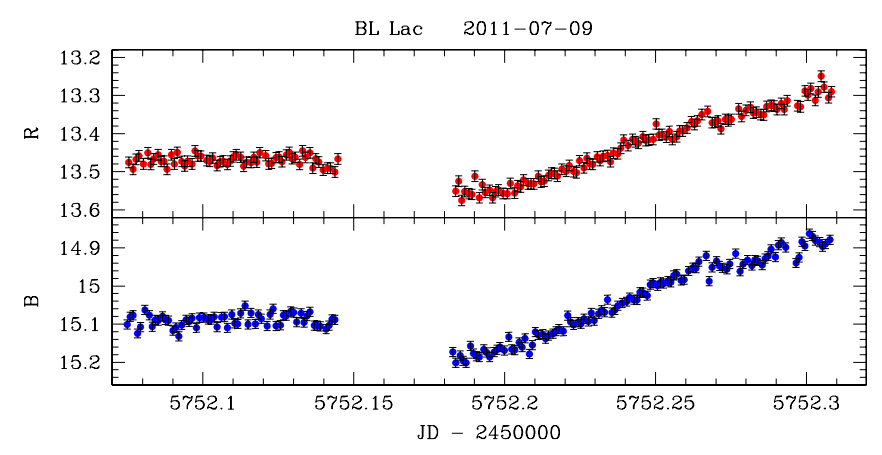

Fig. 9. Similar to Fig. 2, but with time on July 9, 2011 $(\mathrm{JD}=2455752.0749-2455752.3078)$.

(Robinson \& Perez 1990). We calculated the centroid of the DCF, $\tau_{\text {cent }}=\left(\sum_{i} \tau_{i} D C F_{i}\right) /\left(\sum_{i} D C F_{i}\right)$, in order to obtain a more reliable estimate of the time lag (see Raiteri et al. 2003). Monte Carlo simulations of cross-correlation analyses were carried out to check the reliability of the delay. We used the recorded value of the lag to build up the cross-correlation peak distribution (CCPD, Maoz \& Netzer 1989, see Figs. 12 and 13), by repeating the simulation 500 times. As shown in Table 2, $\tau_{\text {cent }}$ is smaller than the corresponding time resolution each day. The results indicated that no measurable time delay longer than the exposure

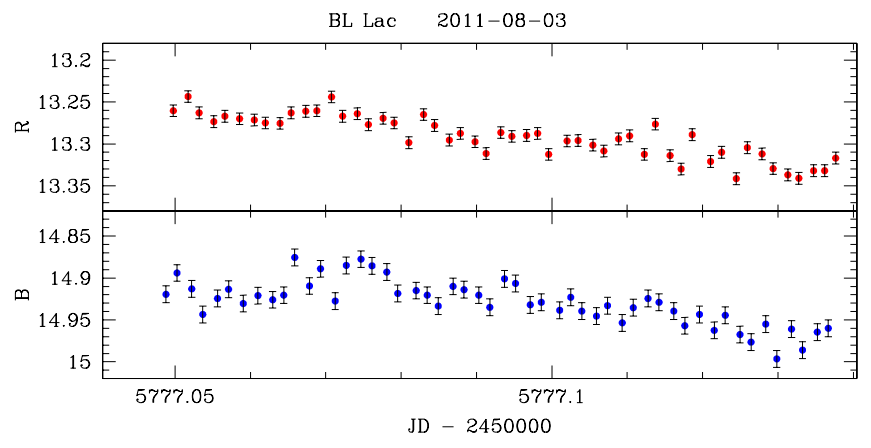

Fig. 10. Similar to Fig. 2, but with time on August 3, 2011 $(\mathrm{JD}=2455777.0485-2455777.1366)$.

interval between $B$ and $R$ bands during the four nights. This coincidence of the $B$ and $R$ band variations suggests that the radiation in both wavebands may be similar in active states and arise from the same region in the jet (Zhai et al. 2011).

\subsection{Colour analysis}

A colour analysis can be an effective tool for exploring the optical variation behaviour of the source and the nature of its emission. Figure 14 shows the $B-R$ colour indices as a function of $R$ magnitudes for BL Lac. The average value is 1.62 mag with a standard deviation of $0.05 \mathrm{mag}$. The colour indices were calculated by selecting both $B$ and $R$ data points with errors not greater than $0.02 \mathrm{mag}$, and by coupling $B$ and $R$ data taken by the same instrument within $5 \mathrm{~min}$.

As shown in Fig. 14, fast flares exhibited a strong bluerwhen-brighter (BWB) trend in inter-night variation (bottom panel), but it is messy in the intra-night variation (top panel). This is consistent with the common trend for blazars when they are in active states (Gu et al. 2006; Wu et al. 2007). According to $\mathrm{Hu}$ et al. (2006), the colour results are not strongly affected by the host galaxy, since it has similar colours to the AGN. In addition, Villata et al. (2002) conclude that colour changes are not related to the host galaxy contribution, but are an intrinsic property of fast flares. Thus, the contribution of the host galaxy was not subtracted from the total flux densities in our analysis.

Colour change can result from two factors: difference in variation steps and amplitudes at different frequencies. Wu et al. (2007) mention that, if the variation at high frequency precedes that at low frequency, a BWB chromatism can be observed. If the fluxes at different frequencies vary simultaneously, but the amplitude is greater at the higher frequency than at the lower one, a BWB chromatism can also be observed. Actually, the colour changes of blazars may be a combination of these two factors. In Sect. 3.2, we do not detect any apparent time lags between the $B$ and $R$ bands. The observed BWB trend should come mainly from the larger amplitudes in the $B$ band. This is confirmed in Sect. 3.1. 


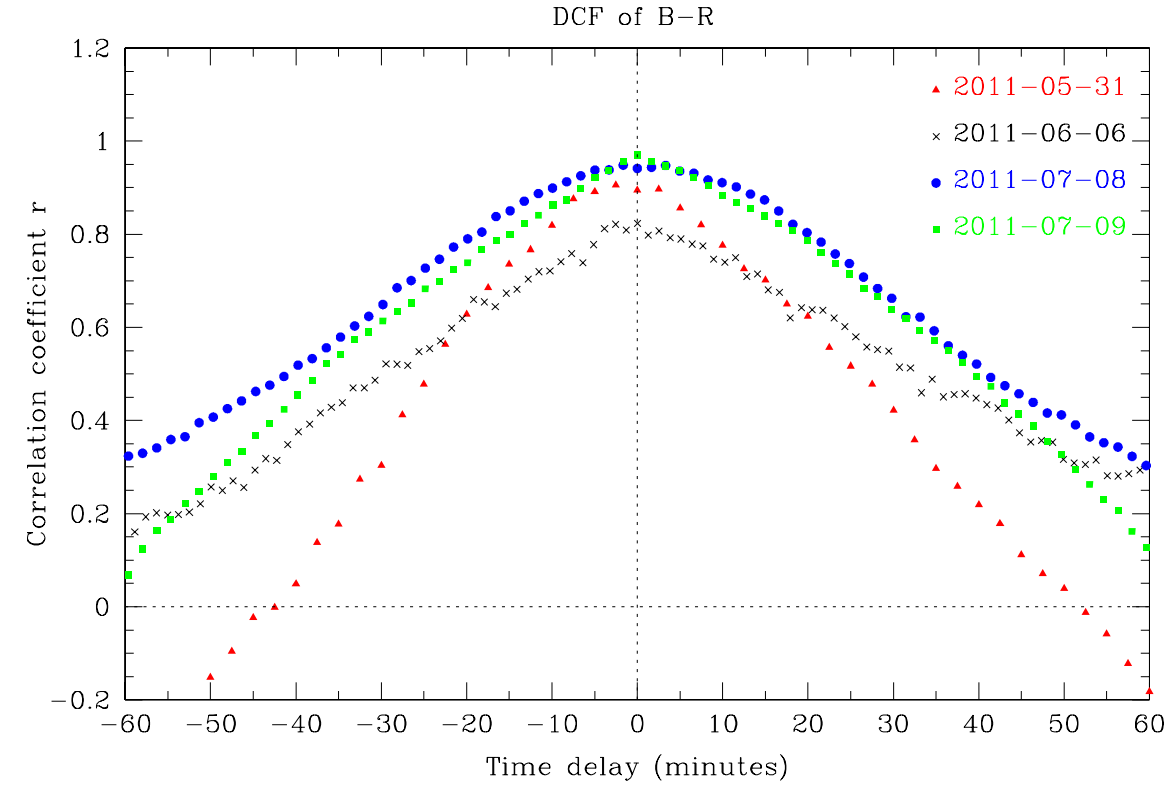

Fig. 11. DCFs between $B$ and $R$ bands light curves on May 31 (red filled triangles), June 6 (black crosses), July 8 (blue filled circles), and July 9 (green filled squares) in 2011. Error bars are omitted for clarity.

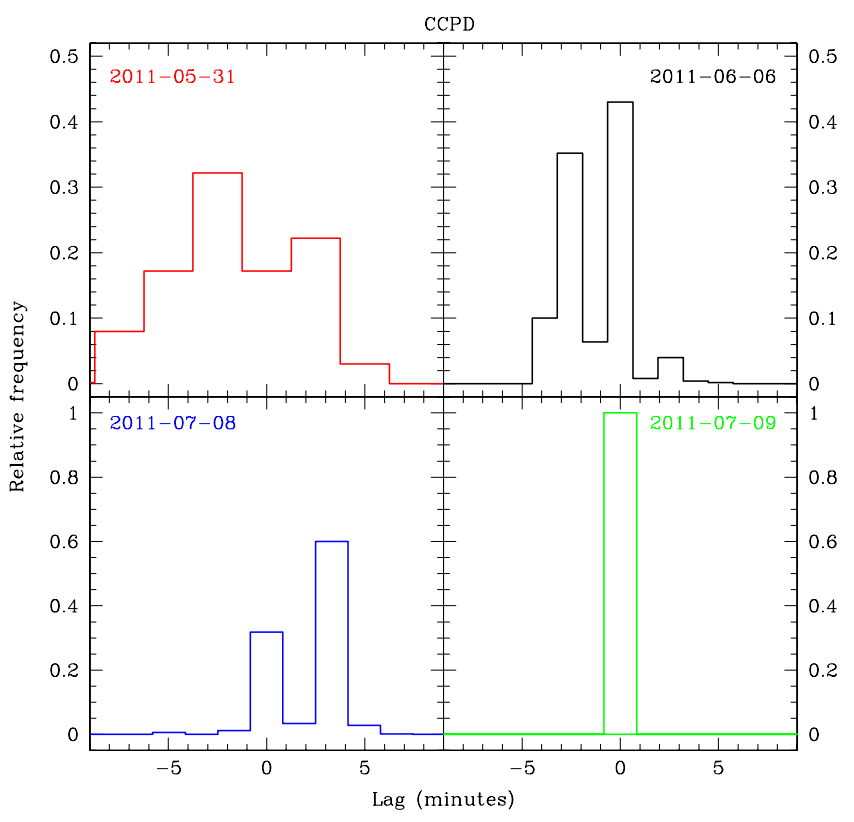

Fig. 12. Cross-correlation peak distributions for $\tau_{\text {peak }}$ on May 31 (red), June 6 (black), July 8 (blue), and July 9 (green) in 2011.

However, the results are ambiguous in the colour versus magnitude diagrams in Fig. 14. When the individual measurements are considered, the diagram is messy only in the top panel, but when the nightly means are considered, there may be a loop in the counterclockwise direction in the bottom panel, as labelled by the arrows. The loop path can be interpreted by a spectral hysteresis, where it can be thought that the variation in one band lags behind that in the other. Our observations did not detect any noticeable time lags between the intra-night variations in the $B$ and $R$ bands, so that the individual measurements do not indicate a loop path. Similar results were reported by Zhai et al. (2011) for the blazar 3C 454.3. However, the loop paths described by the nightly means may suggest that BL Lac has a spectral hysteresis in its inter-night variations (Wu et al. 2007). The intra-night variations in active states can be determined by a "strongly-chromatic" short-term component, which may

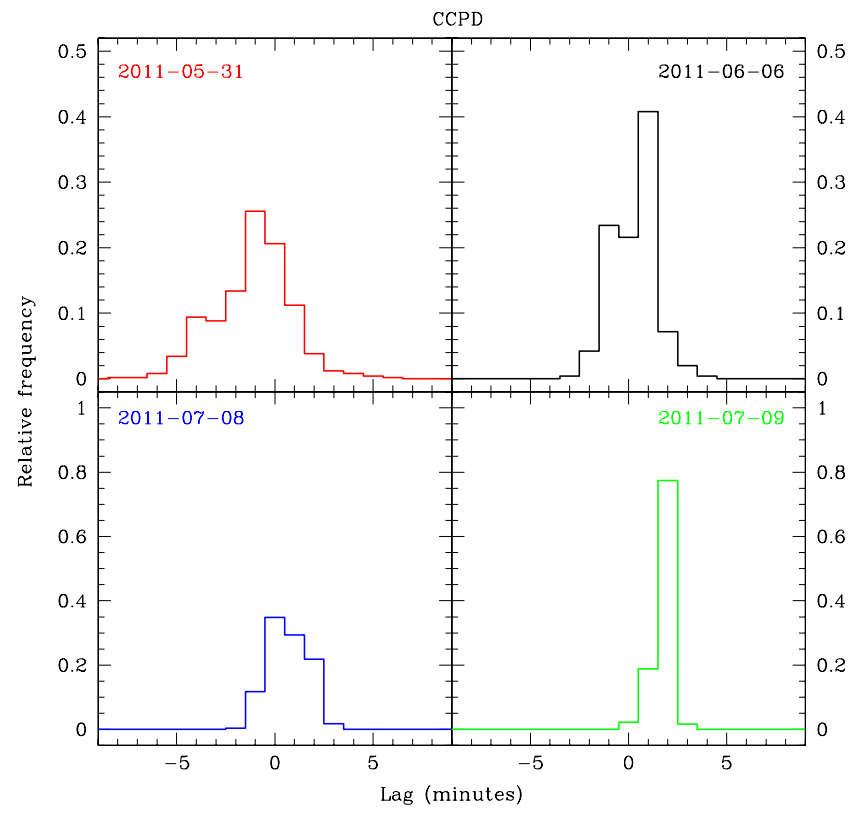

Fig. 13. Cross-correlation peak distributions for $\tau_{\text {cent }}$ on May 31 (red), June 6 (black), July 8 (blue), and July 9 (green) in 2011.

be caused by intrinsic phenomena, such as particle acceleration from shock-in-jet events (Villata et al. 2004).

\section{Conclusions}

We have presented our simultaneous $(\Delta t \leqslant 2.5 \mathrm{~min}$ in most cases) optical photometric datasets of BL Lac collected in the $B$ and $R$ bands by the TNT during the 2011 outburst. The main results can be summarized as follows.

- We detected an optical outburst in May-August 2011. The source displayed strong variability with amplitudes $\triangle B=$ $1.46 \mathrm{mag}$ and $\Delta R=1.31 \mathrm{mag}$ during our observing season. The brightest level ( $B=14.48 \mathrm{mag}, R=12.89 \mathrm{mag}$ ) in our observation was detected in May 27. A rapid brightening with a rising rate of $\sim 0.12$ mag per hour was found in 


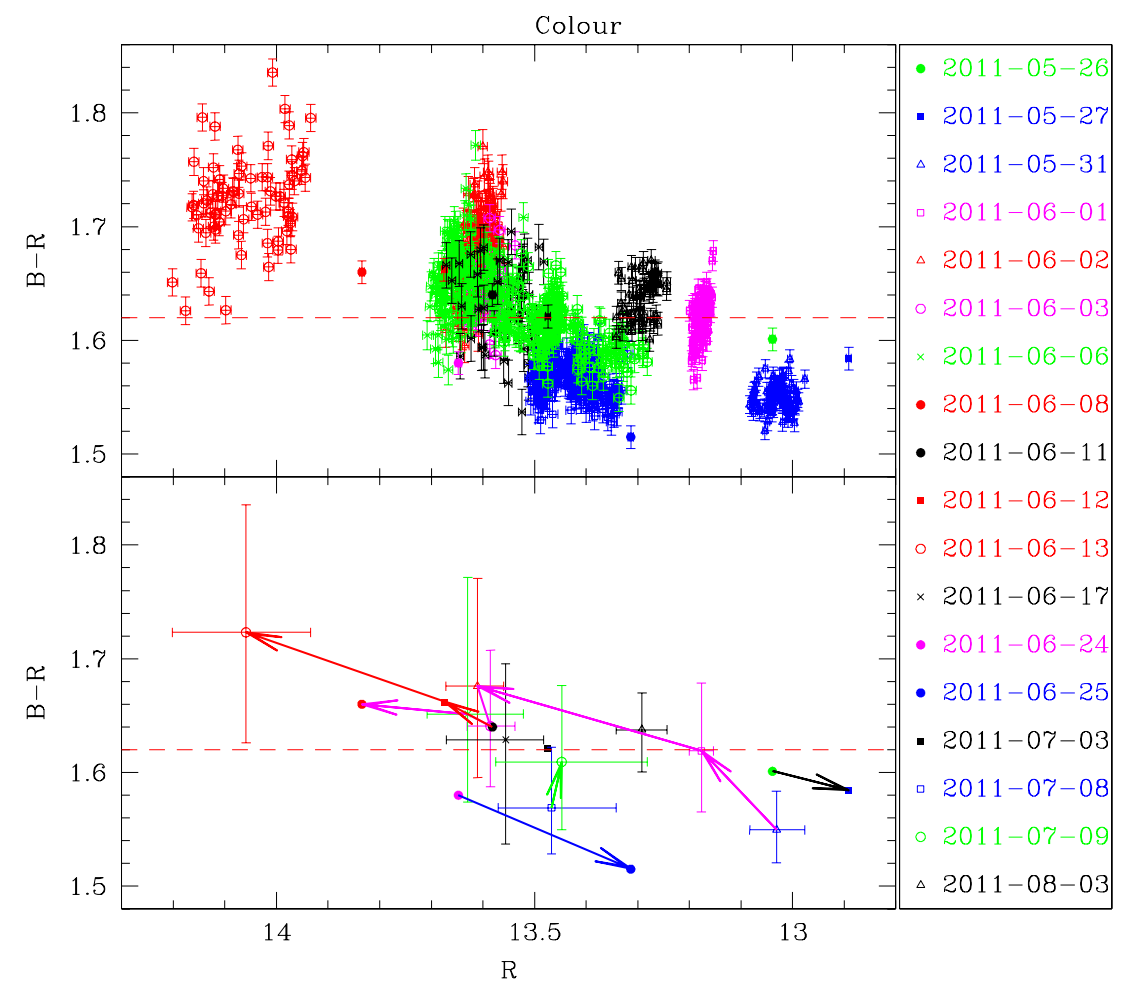

Fig. 14. The $B-R$ colour indices as a function of $R$ magnitudes during the 18 nights in the right hand list. The top panel contains 1050 colour index points. To clarify the colour variability, in the bottom panel the $\overline{B-R}$ and $\bar{R}$ values in each night are reported instead of the dense points, while the bars indicate the variable range of $B-R$ and $R$. The arrows indicate the time sequence. In both panels, the red dashed lines indicate the average $B-R$ colour index (1.62).
July 9. The outbursts may be possible induced by both intrinsic mechanisms (shock-in-jet model) and geometrical effects (Doppler factor).

- Our data, which are characterized by high temporal resolution and simultaneous measurements, show no time delay exceeding $2.5 \mathrm{~min}$ between variations in the $B$ and $R$ bands. Cross-correlation analysis suggests that the radiation in both wavebands may have similar natures in active states, and arise from the same region in the jet.

- We detected a bluer-when-brighter trend in the outburst, and the colour variability processes are apparently complex. These trends may be interpreted as the interplay of a "strongly chromatic" intra-night component and a "mildly chromatic" inter-night component.

We conclude that, both the amplitude of variability and the variation rate are greater at the shorter wavelength. Different variation amplitudes in different bands will lead to colour changes. Our observations did not detect any significant intra-night time lag between $B$ and $R$ bands. The $\tau_{\text {peak }}$ should be treated with caution, since it is close to the temporal resolution of observation. Thus, the present extension of the BL Lac optical dataset leads to a more refined scenario. However, additional multifrequency monitoring is still needed to explore the nature of BL Lac in detail.

Acknowledgements. We are grateful to the referee for valuable comments and detailed suggestions that have been adopted to improve this paper very much. We owe thanks to the TNT time allocation committee for arranging ToO observations. The authors are grateful to Chunlan Lu and Liang Ma for their night assistant work. We are thankful to Pingping Liu (Chinese Academy of Sciences, China) for stimulating discussions that helped us to improve this paper. This work was supported by the Chinese Natural Science Foundation (No.10803008). It was also supported by the National Basic Research Programme of China - 973 Programme (2009CB824800).

\section{References}

Capetti, A., Raiteri, C. M., \& Buttiglione, S. 2010, A\&A, 516, A59

Carrasco, L., Carraminana, A., Escobedo, G., et al. 2011, The Astronomer's Telegram, 3375, 1

Cutini, S. 2011, The Astronomer's Telegram, 3368, 1

Dreissigacker, O., \& Camenzind, M. 1996, Blazar Continuum Variability, 110, 377

Edelson, R. A., \& Krolik, J. H. 1988, ApJ, 333, 646

Fan, J. H., Qian, B. C., \& Tao, J. 2001, A\&A, 369, 758

Gasparrini, D. 2011, The Astronomer's Telegram, 3462,

Gu, M. F., Lee, C.-U., Pak, S., Yim, H. S., \& Fletcher, A. B. 2006, A\&A, 450, 39

Gupta, A. C., \& Joshi, U. C. 2005, A\&A, 440, 855

Hu, S. M., Wu, J. H., Zhao, G., \& Zhou, X. 2006, MNRAS, 373, 209

Hufnagel, B. R., \& Bregman, J. N. 1992, ApJ, 386, 473

Larionov, V. M., Larionova, E. G., Efimova, N. V., Villata, M., \& Raiteri, C. M. 2011, The Astronomer's Telegram, 3371, 1

Maoz, D., \& Netzer, H. 1989, MNRAS, 236, 21

Marscher, A. P. 1996, Blazar Continuum Variability, 110, 248

Miller, J. S., \& Hawley, S. A. 1977, ApJ, 212, L47

Ong, R. A. 2011, The Astronomer's Telegram, 3459, 1

Peterson, B. M., Wanders, I., Horne, K., et al. 1998, PASP, 110, 660

Raiteri, C. M., Villata, M., Tosti, G., et al. 2003, A\&A, 402, 151

Raiteri, C. M., Villata, M., Capetti, A., et al. 2009, A\&A, 507, 769

Raiteri, C. M., Villata, M., Bruschini, L., et al. 2010, A\&A, 524, A43

Robinson, A., \& Perez, E. 1990, MNRAS, 244, 138

Schneider, P., \& Weiss, A. 1987, A\&A, 171, 49

Shen, B. S. P., \& Usher, P. D. 1970, Nature, 228, 1070

Smith, P. S., Balonek, T. J., Heckert, P. A., Elston, R., \& Schmidt, G. D. 1985, AJ, 90, 1184

Villata, M., \& Raiteri, C. M. 1999, A\&A, 347, 30

Villata, M., Raiteri, C. M., Kurtanidze, O. M., et al. 2002, A\&A, 390, 407 Villata, M., Raiteri, C. M., Kurtanidze, O. M., et al. 2004, A\&A, 421, 103

Villata, M., Raiteri, C. M., Larionov, V. M., et al. 2009, A\&A, 501, 455

Wiita, P. J. 1996, Blazar Continuum Variability, 110, 42

Wu, J., Zhou, X., Ma, J., et al. 2007, AJ, 133, 1599

Zhai, M., Zheng, W. K., \& Wei, J. Y. 2011, A\&A, 531, A90 